Note on the $q$-Stancu-Schurer operator

Carmen Violeta Muraru 


\title{
NOTE ON THE $q$-STANCU-SCHURER OPERATOR
}

\author{
CARMEN VIOLETA MURARU
}

Received December 19, 2011

\begin{abstract}
In this paper, we introduce a generalization of the Stancu- Schurer operators based on q-integers and get a Bohman-Korovkin type approximation theorem of these operators. We also compute the rate of convergence by using the first modulus of smoothness and give some numerical result of operators based on Matlab algorithms.
\end{abstract}

2000 Mathematics Subject Classification: 41A36

Keywords: q-integers, positive linear operator, Bernstein operator, modulus of continuity

\section{PReliminaries}

After the construction of q-Bernstein operators by Lupas [13] in 1987 and then by Phillips [12] many authors studied new classes of q-generalized operators. To show the extend of this research direction, we mention in the following some achievements in this field. In [1] O. Agratini introduced a new class of q-Bernstein-type operators which fix certain polynomials and studied the limit of iterates of Lupas q-analogue of the Bernstein operators. In [3] Aral and Doğru obtained the uniform approximation of q-Bleimann- Butzer-Hahn (BBH) operators and in [5] O. Doğru and V. Gupta studied the monotonicity properties and the Voronovskaja type asymptotic estimate of these operators. See also the recent paper [2].

T. Trif [11] investigated Meyer-Kőnig and Zeller (MKZ) operators based on q-integers. Some approximation properties of q-MKZ operators were investigated by W. Heping in [10]. O. Doğru and O. Duman introduced also a new generalization of MeyerKônig and Zeller operators and studied some statistical approximation properties in [4]. O. Doğru and Gupta [6] constructed a q-type generalization of Meyer-Kőnig and Zeller operators in bivariate case.

A new q-generalization of Meyer-Kőnig and Zeller type operators was constructed by Doğru and Muraru for improve the rate of convergence, see [7].

Gupta and Heping introduced a q-analoque of Bernstein-Durrmeyer operators in [9] and in 2009 Gupta and Finta [8] studied some local and global approximation properties for q-Durrmeyer operators. In [10] Gupta and Radu constructed a q-analoque of 
Baskakov-Kantorovich operators and investigated their weighted statistical approximation properties.

For our study we remind first some elements of q-Calculus. For any fixed real number $q>0$, the q-integer $[k]_{q}$, for $k \in N$ is defined by

$$
[k]_{q}= \begin{cases}\left(1-q^{k}\right) /(1-q), & q \neq 1 \\ k, & q=1\end{cases}
$$

The q-factorial integer and q-binomial coefficients are :

$$
\begin{aligned}
& {[k]_{q} != \begin{cases}{[k][k-1] \ldots[1],} & k=1,2, \ldots \\
1, & k=0\end{cases} } \\
& {\left[\begin{array}{c}
n \\
k
\end{array}\right]_{q}=\frac{[n]_{q} !}{[k]_{q} ![n-k]_{q} !} \quad(n \geq k \geq 0)}
\end{aligned}
$$

The q-analoque of $(x-a)^{n}$ is the polynomial

$$
(x-a)_{q}^{n}= \begin{cases}1, & \text { if } n=0 \\ (x-a)(x-q a) \ldots\left(x-q^{n-1} a\right), & \text { if } n \geq 1\end{cases}
$$

As usual, we note with $C([a, b])$, the space of all real valued continuous functions defined on $[a, b]$. The space is endowed with usual norm $\|\cdot\|$ given by $\|f\|=\sup _{x \in[a, b]}|f(x)|$.

Let $p \in N$ be fixed. In 1962 Schurer introduced and studied the Bernstein-Schurer operators $\tilde{B}_{m, p}: C([0, p+1]) \rightarrow C([0,1])$ defined for any $m \in N$ and any function $f \in C([0, p+1])$ as follows

$$
\tilde{B}_{m, p}(f ; x)=\sum_{k=0}^{m+p}\left(\begin{array}{c}
m+p \\
k
\end{array}\right) x^{k}(1-x)^{m+p-k} f\left(\frac{k}{m}\right)
$$

where $\tilde{p}_{m, k}(x)=\left(\begin{array}{c}m+p \\ k\end{array}\right) x^{k}(1-x)^{m+p-k}$ are the fundamental Schurer's polynomials.

Let $\mathrm{p}$ be a non-negative integer and let $\alpha, \beta$ be a real parameters satisfying the conditions $0 \leq \alpha \leq \beta$.

The Schurer-Stancu operator $\tilde{S}_{m, p}^{(\alpha, \beta)}: C([0,1+p]) \rightarrow C([0,1])$ is defined for any $m \in N^{*}$, any $f \in C([0,1+p])$ and any $x \in[0,1]$ by

$$
\left(\tilde{S}_{m, p}^{(\alpha, \beta)} f\right)(x)=\sum_{k=0}^{m+p} \tilde{p}_{m, k}(x) f\left(\frac{k+\alpha}{m+\beta}\right) .
$$

Lemma 1. For any $x \in[0,1+p]$ the following relations
(1) $\left(\tilde{S}_{m, p}^{(\alpha, \beta)} e_{0}\right)(x)=1$
(2) $\left(\tilde{S}_{m, p}^{(\alpha, \beta)} e_{1}\right)(x)=\frac{m+p}{m+\beta} x+\frac{\alpha}{m+\beta}$ 
(3) $\left(\tilde{S}_{m, p}^{(\alpha, \beta)} e_{2}\right)(x)=$

$$
=\frac{1}{(m+\beta)^{2}}\left\{(m+p)^{2} x^{2}+(m+p) x(1-x)+2 \alpha(m+p) x+\alpha^{2}\right\}
$$

hold where $e_{j}(x)=x^{j}(j \in N)$ denotes like usually test monomials.

Our aim is to introduce a q-analogue of the above operators. We investigate the approximation properties of this class and we estimate the rate of convergence by using modulus of continuity .

\section{APPROXIMATION PROPERTIES OF $q$-SCHURER-STANCU OPERATORS}

We will consider in the following that $q \in(0,1)$.

For any $m \in N$, $p$ a fixed non negative number and $\alpha, \beta$ a real parameters satisfying the conditions $0 \leq \alpha \leq \beta$, we construct the class of generalized q-Schurer-Stancu operators $\left(S_{m, p, q}^{(\alpha, \beta)} f\right)(x): C[0,1+p] \rightarrow C[0,1]$ as follows:

$$
\left(S_{m, p, q}^{(\alpha, \beta)} f\right)(x)=\sum_{k=0}^{m+p}\left[\begin{array}{c}
m+p \\
k
\end{array}\right]_{q} x^{k} \prod_{s=0}^{m+p-k-1}\left(1-q^{s} x\right) f\left(\frac{[k+\alpha]_{q}}{[m+\beta]_{q}}\right)
$$

for any $x \in[0,1]$.

Lemma 2. For the polynomials defined above the following properties hold

(1) $\left(\mathrm{S}_{m, p, q}^{(\alpha, \beta)} e_{0}\right)(x)=1$

(2) $\left(S_{m, p, q}^{(\alpha, \beta)} e_{1}\right)(x)=\frac{[\alpha]_{q}}{[m+\beta]_{q}}+\frac{q^{\alpha}[m+p]_{q}}{[m+\beta]_{q}} x$

(3) $\left(S_{m, p, q}^{(\alpha, \beta)} e_{2}\right)(x)=$

$$
=\frac{[\alpha]_{q}^{2}}{[m+\beta]_{q}^{2}}+\frac{q^{2 \alpha}[m+p]_{q}^{2}}{[m+\beta]_{q}^{2}} x^{2}+\frac{2 q^{\alpha}[\alpha]_{q}[m+p]_{q} x}{[m+\beta]_{q}^{2}}+\frac{q^{2 \alpha}[m+p]_{q}}{[m+\beta]_{q}^{2}} x(1-x) .
$$

Proof. (1) We use the known identity

$$
\sum_{k=0}^{n}\left[\begin{array}{c}
n \\
k
\end{array}\right]_{q} x^{k}(1-x)_{q}^{n-k}=1
$$

which can be proved by induction with respect to $\mathrm{n}$. The left hand side represents $\left(B_{n, q} e_{0}\right)(x)$ where $B_{n, q}$ is the q-analogue of Bernstein operator introduced by Phillips.

If we choose $n=m+p$, taking into account that

$$
(1-x)^{m+p-k}=\prod_{s=0}^{m+p-k-1}\left(1-q^{s} x\right)
$$

we get

$$
\sum_{k=0}^{m+p}\left[\begin{array}{c}
m+p \\
k
\end{array}\right]_{q} x^{k} \prod_{s=0}^{m+p-k-1}\left(1-q^{s} x\right)=1
$$


Consequently we obtain

$$
\left(\mathrm{S}_{m, p, q}^{(\alpha, \beta)} e_{0}\right)(x)=\sum_{k=0}^{m+p}\left[\begin{array}{c}
m+p \\
k
\end{array}\right] x \prod_{s=0}^{m+p-k-1}\left(1-q^{s} x\right)=1 .
$$

(2) $\left(S_{m, p, q}^{(\alpha, \beta)} e_{1}\right)(x)=$

$$
\begin{aligned}
& =\sum_{k=0}^{m+p}\left[\begin{array}{c}
m+p \\
k
\end{array}\right]_{q} x^{k} \prod_{s=0}^{m+p-k-1}\left(1-q^{s} x\right) \frac{[k+\alpha]_{q}}{[m+\beta]_{q}} \\
& =\frac{1}{[m+\beta]_{q}} \sum_{k=0}^{m+p} \frac{[m+p]_{q} ![k+\alpha]_{q}}{[k]_{q} ![m+p-k]_{q} !} x^{k} \prod_{s=0}^{m+p-k-1}\left(1-q^{s} x\right) \\
& =\frac{1}{[m+\beta]_{q}} \sum_{k=0}^{m+p} \frac{[m+p]_{q} !\left([\alpha]_{q}+q^{\alpha}[k]_{q}\right)}{[k]_{q} ![m+p-k]_{q} !} x^{k} \prod_{s=0}^{m+p-k-1}\left(1-q^{s} x\right) \\
& =\frac{[\alpha]_{q}}{[m+\beta]_{q}}+\frac{q^{\alpha}[m+p]_{q}}{[m+\beta]_{q}} x \sum_{k=1}^{m+p} \frac{[m+p-1]_{q} !}{[k-1]_{q} ![m+p-k]_{q} !} x^{k-1} \prod\left(1-q^{s} x\right) \\
& =\frac{[\alpha]_{q}}{[m+\beta]_{q}}+\frac{q^{\alpha}[m+p]_{q}}{[m+\beta]_{q}} x .
\end{aligned}
$$

We choose $q$ a sequence that $q_{m}^{\alpha} \rightarrow 1$, for $m \rightarrow \infty$ and we have

$$
\left(S_{m, p, q}^{(\alpha, \beta)} e_{1}\right)(x) \rightarrow x
$$

(3) $\left(S_{m, p, q}^{(\alpha, \beta)} e_{2}\right)(x)=$

$$
\begin{aligned}
= & \sum_{k=0}^{m+p}\left[\begin{array}{c}
m+p \\
k
\end{array}\right]_{q} x^{k} \prod_{s=0}^{m+p-k-1}\left(1-q^{s} x\right) \frac{[k+\alpha]_{q}^{2}}{[m+\beta]_{q}^{2}} \\
= & \frac{1}{[m+\beta]_{q}^{2}} \sum_{k=0}^{m+p} \frac{[m+p]_{q} ![k+\alpha]_{q}[k+\alpha]_{q}}{[k]_{q} ![m+p-k]_{q} !} x^{k} \prod_{s=0}^{m+p-k-1}\left(1-q^{s} x\right) \\
= & \frac{1}{[m+\beta]_{q}^{2}} \sum_{k=0}^{m+p} \frac{[m+p]_{q} !\left([\alpha]_{q}+q^{\alpha}[k]_{q}\right)[k+\alpha]_{q}}{[k]_{q} ![m+p-k]_{q} !} x^{k} \prod_{s=0}^{m+p-k-1}\left(1-q^{s} x\right) \\
= & \frac{1}{[m+\beta]_{q}^{2}} \sum_{k=0}^{m+p} \frac{[m+p]_{q} ![\alpha]_{q}[k+\alpha]_{q}}{[m+p-k]_{q} ![k]_{q} !} x^{k} \prod_{s=0}^{m+p-k-1}\left(1-q^{s} x\right) \\
& +\frac{q^{\alpha}}{[m+\beta]_{q}^{2}} \sum_{k=0}^{m+p} \frac{[k]_{q}\left([\alpha]_{q}+q^{\alpha}[k]_{q}\right)[m+p]_{q} !}{[m+p-k]_{q} ![k]_{q} !} x^{k} \prod_{s=0}^{m+p-k-1}\left(1-q^{s} x\right)
\end{aligned}
$$




$$
\begin{aligned}
& =\frac{[\alpha]}{[m+\beta]_{q}^{2}} \sum_{k=0}^{m+p} \frac{[m+p]_{q} !\left([\alpha]_{q}+q^{\alpha}[k]_{q}\right)}{[m+p-k]_{q} ![k]_{q} !} x^{k} \prod_{s=0}^{m+p-k-1}\left(1-q^{s} x\right) \\
& +\frac{q^{\alpha}[\alpha]_{q}}{[m+\beta]_{q}^{2}} \sum_{k=0}^{m+p} \frac{[k]_{q}[m+p]_{q} !}{[m+p-k]_{q} ![k]_{q} !} x^{k} \prod_{s=0}^{m+p-k-1}\left(1-q^{s} x\right) \\
& +\frac{q^{\alpha}[\alpha]_{q}}{[m+\beta]_{q}^{2}} \sum_{k=0}^{m+p} \frac{[k]_{q}^{2}[m+p]_{q} !}{[m+p-k]_{q} ![k]_{q} !} x^{k} \prod_{s=0}^{m+p-k-1}\left(1-q^{s} x\right) \\
& =\frac{[\alpha]_{q}}{[m+\beta]_{q}^{2}}\left([\alpha]_{q}+q^{\alpha}[m+p]_{q} x\right)+\frac{q^{\alpha}[\alpha][m+p]_{q} x}{[m+\beta]_{q}^{2}} \\
& +\frac{q^{2 \alpha}}{[m+\beta]_{q}^{2}} \sum_{k=0}^{m+p} \frac{\left(q[k-1]_{q}+1\right)[k]_{q}[m+p]_{q} !}{[m+p-k]_{q} ![k]_{q} !} x^{k} \prod_{s=0}^{m+p-k-1}\left(1-q^{s} x\right) \\
& =\frac{[\alpha]_{q}}{[m+\beta]_{q}^{2}}\left([\alpha]_{q}+q^{\alpha}[m+p]_{q} x\right) \\
& +\frac{q^{\alpha}[\alpha][m+p]_{q} x}{[m+\beta]_{q}^{2}}+\frac{q^{2 \alpha}}{[m+\beta]_{q}^{2}} \sum_{k=0}^{m+p} \frac{\left(q[k-1]_{q}[k]_{q}[m+p]_{q} !\right)}{[m+p-k]_{q} ![k]_{q} !} x \prod_{s=0}^{m+p-k-1}\left(1-q^{s} x\right) \\
& +\frac{q^{2 \alpha}}{[m+\beta]_{q}^{2}} \sum_{k=0}^{m+p} \frac{[k]_{q}[m+p]_{q} !}{[m+p-k]_{q} ![k]_{q} !} x^{k} \prod_{s=0}^{m+p-k-1}\left(1-q^{s} x\right) \\
& =\frac{[\alpha]}{[m+\beta]_{q}^{2}}\left([\alpha]_{q}+q^{\alpha}[m+p]_{q} x\right)+\frac{q^{\alpha}[\alpha]_{q}[m+p]_{q} x}{[m+\beta]_{q}^{2}} \\
& +\frac{x^{2}[m+p]_{q}[m+p-1]_{q} q^{2 \alpha+1}}{[m+\beta]_{q}^{2}}+\frac{q^{2 \alpha}[m+p]_{q}}{[m+\beta]_{q}^{2}} x \\
& =\frac{[\alpha]^{2}}{[m+\beta]_{q}^{2}}+\frac{q^{2 \alpha}[m+p]_{q}^{2}}{[m+\beta]_{q}^{2}} x^{2}+\frac{2 q^{\alpha}[\alpha][m+p]_{q} x}{[m+\beta]_{q}^{2}}+\frac{q^{2 \alpha}[m+p]_{q}}{[m+\beta]_{q}^{2}} x(1-x) .
\end{aligned}
$$

We used that $[m+p-1] q=[m+p]-1$.

\section{ESTIMATION OF CONVERGENCE}

We will estimate the rate of convergence in terms of modulus of continuity. Let $f \in C([0, b])$. The modulus of continuity of $f$ denoted by $\omega_{f}(\delta)$ gives the maximum oscillation of $f$ in any interval of length not exceeding $\delta>0$ and it is given by the relation

$$
\omega_{f}(\delta)=\sup _{|y-x| \leq \delta}|f(y)-f(x)|, \quad x, y \in[0, b]
$$


It is well-known that for $f \in C([0, b])$

$$
\lim _{\delta \rightarrow 0} \omega_{f}(\delta)=0,
$$

and for any $\delta>0$

$$
|f(y)-f(x)| \leq \omega_{f}(\delta)\left(\frac{|y-x|}{\delta}+1\right) .
$$

The next result is based on Popoviciu technique for the considered operators in the terms of first order modulus of continuity.

Theorem 1. If $f \in C([0,1+p])$ then

$$
\left|\left(S_{m, p, q}^{(\alpha, \beta)} f\right)(x)-f(x)\right| \leq 2 \omega_{f}\left(\delta_{m}\right)
$$

takes place, when

$$
\delta_{m}=\frac{1}{\sqrt{[m]_{q}}}\left(\frac{p}{1-q^{m}}+\frac{1}{2} \sqrt{\frac{1-q^{m+p}}{1-q^{m}}}\right), \quad q \in(0,1) .
$$

Proof. Since $\frac{[m+p]_{q} !}{[m+p-k]_{q} ![k]_{q} !}>0, \quad x^{k}>0, \quad \prod_{s=0}^{m+p-k-1}\left(1-q^{s} x\right)>0$ we have

$$
\begin{aligned}
& \left|\left(S_{m, p, q}^{(\alpha, \beta)} f\right)(x)-f(x)\right| \leq \\
& \leq \sum_{k=0}^{m+p}\left|f\left(\frac{[k+\alpha]_{q}}{[m+p]_{q}}\right)-f(x)\right| \frac{[m+p]_{q} !}{[m+p-k]_{q} ![k]_{q} !} x^{k} \prod_{s=0}^{m+p-k-1}\left(1-q^{s} x\right)
\end{aligned}
$$

Using (3.1) we have

$$
\left.\begin{array}{l}
\left|\left(S_{m, p, q}^{(\alpha, \beta)} f\right)(x)-f(x)\right| \leq \\
\leq \omega_{f}(\delta)\left\{\frac{1}{\delta} \sum_{k=0}^{m+p}\left|\frac{[k+\alpha]_{q}}{[m+p]_{q}}-x\right| \frac{[m+p]_{q} !}{[m+p-k]_{q} ![k]_{q} !} x^{k} \prod_{s=0}^{m+p-k}\left(1-q^{s} x\right)\right. \\
\left.\quad+\sum_{k=0}^{m+p} \frac{[m+p]_{q} !}{[m+p-k]_{q} ![k]_{q} !} x^{k} \prod_{s=0}^{m+p-k}\left(1-q^{s} x\right)\right\} \\
=\omega_{f}(\delta)\left\{\frac{1}{\delta} \sum_{k=0}^{m+p}\left|\frac{[k+\alpha]_{q}}{[m+p]_{q}}-x\right| \frac{[m+p]_{q} !}{[m+p-k]_{q} ![k]_{q} !} x^{k}\right. \\
\cdot \prod_{s=0}^{m+p-k}\left(1-q^{s} x\right)+\left(S_{m, p, q}^{(\alpha, \beta)} e_{0}\right)(x)
\end{array}\right\}
$$


Using Cauchy-Schwartz inequality we have

$$
\begin{aligned}
& \left|\left(S_{m, p, q}^{(\alpha, \beta)} f\right)(x)-f(x)\right| \leq \\
& \leq \omega_{f}(\delta)\left\{\frac{1}{\delta}\left(\sum_{k=0}^{m+p}\left(\frac{[k+\alpha]_{q}}{[m+p]_{q}}-x\right)^{2} \frac{[m+p]_{q} !}{[m+p-k]_{q} ![k]_{q} !} x^{k} \prod_{s=0}^{m+p-k}\left(1-q^{s} x\right)\right)^{1 / 2}+1\right\} \\
& \leq \omega_{f}(\delta)\left\{\frac{1}{\delta}\left(\left(S_{m, p, q}^{(\alpha, \beta)} e_{2}\right)(x)-2 x\left(S_{m, p, q}^{(\alpha, \beta)} e_{1}\right)(x)+x^{2}\left(S_{m, p, q}^{(\alpha, \beta)} e_{0}\right)(x)\right)^{1 / 2}+1\right\}
\end{aligned}
$$

Finally, taking into account that $0<\mathrm{x}<1$

$$
\begin{aligned}
& \left|\left(S_{m, p, q}^{(\alpha, \beta)} f\right)(x)-f(x)\right| \leq \\
& \leq \omega_{f}(\delta)\left\{\frac { 1 } { \delta } \left(\frac{[\alpha]^{2}}{[m+\beta]^{2}}+\frac{q^{2 \alpha}[m+p]^{2}}{[m+\beta]^{2}} x^{2}+\frac{2 q^{\alpha}[\alpha][m+p] x}{[m+\beta]^{2}}+\frac{q^{2 \alpha}[m+p]}{[m+\beta]^{2}} x(1-x)\right.\right. \\
& \left.\quad-2 x\left(\frac{[\alpha]}{[m+\beta]}+x q^{\alpha} \frac{[m+p]}{[m+\beta]}\right)+x^{2}\right)^{1 / 2}+1 \\
& =\omega_{f}(\delta)\left\{\frac { 1 } { \delta } \left(x^{2}\left(q^{\alpha} \frac{[m+p]_{q}}{[m+\beta]_{q}}-1\right)^{2}+q^{2 \alpha} x(1-x) \frac{[m+p]_{q}}{[m+\beta]_{q}^{2}}\right.\right. \\
& \left.\left.\quad+2 x \frac{[\alpha]}{[m+\beta]}\left(q^{\alpha} \frac{[m+p]}{[m+\beta]}-1\right)+\frac{[\alpha]^{2}}{[m+\beta]^{2}}\right)^{1 / 2}+1\right\}
\end{aligned}
$$

Using the inequality $\left(a^{2}+b^{2}\right)^{1 / 2} \leq|a|+|b|$ and that $x \in[0,1], x(1-x) \leq 1 / 4$ and $q^{\alpha} \leq 1$, the relation (3.3) becomes

$$
\begin{aligned}
& \left|\left(S_{m, p, q}^{(\alpha, \beta)} f\right)(x)-f(x)\right| \leq \\
& \leq \omega_{f}(\delta)\left\{\frac { 1 } { \delta } \left(\left(q^{\alpha} \frac{[m+p]_{q}}{[m+\beta]_{q}}-1\right)^{2}+\frac{1}{4} q^{2 \alpha} \frac{[m+p]_{q}}{[m+\beta]_{q}^{2}}\right.\right. \\
& \left.\left.+2 \frac{[\alpha]_{q}}{[m+\beta]_{q}}\left(q^{\alpha} \frac{[m+p]_{q}}{[m+\beta]_{q}}-1\right)+\frac{[\alpha]_{q}^{2}}{[m+\beta]_{q}^{2}}\right)^{1 / 2}+1\right\} \\
& \leq \omega_{f}(\delta)\left\{\frac{1}{\delta}\left(\left(q^{\alpha} \frac{[m+p]_{q}}{[m+\beta]_{q}}-1+\frac{[\alpha]_{q}}{[m+\beta]_{q}}\right)^{2}+\frac{1}{4} q^{2 \alpha} \frac{[m+p]_{q}}{[m+\beta]_{q}^{2}}\right)^{1 / 2}+1\right\} \\
& \leq \omega_{f}(\delta)\left\{\frac{1}{\delta}\left(\left|q^{\alpha} \frac{[m+p]_{q}}{[m+\beta]_{q}}-1+\frac{[\alpha]}{[m+\beta]_{q}}\right|+\frac{1}{2} q^{\alpha} \frac{\sqrt{[m+p]_{q}}}{[m+\beta]_{q}}\right)+1\right\} \\
& \leq \omega_{f}(\delta)\left\{\frac{1}{\delta}\left(\frac{q^{m}}{1-q^{m+\beta}}\left|q^{\beta}-q^{\alpha+p}\right|+\frac{1}{2} q^{\alpha} \frac{\sqrt{1-q} \sqrt{1-q^{m+p}}}{1-q^{m+\beta}}\right)+1\right\}
\end{aligned}
$$




$$
\leq \omega_{f}(\delta)\left\{\frac{1}{\delta}\left(\frac{q^{m}}{1-q^{m}}+\frac{1}{2} \frac{\sqrt{1-q} \sqrt{1-q^{m+p}}}{1-q^{m}}\right)+1\right\}
$$

From $\frac{q^{m}\left(1-q^{p}\right)}{1-q^{m}} \leq \frac{p}{\sqrt{[m]_{q}}}$ it follows that

$$
\frac{q^{m}}{1-q^{m}} \leq \frac{p}{\left(1-q^{p}\right) \sqrt{[m]_{q}}} \leq \frac{p}{\left(1-q^{m}\right) \sqrt{[m]_{q}}} \quad(p<m) .
$$

The relation (3.4) becomes

$$
\begin{aligned}
& \left|\left(S_{m, p, q}^{(\alpha, \beta)} f\right)(x)-f(x)\right| \leq \\
& \leq \omega_{f}(\delta)\left\{\frac{1}{\delta}\left(\frac{p}{\left(1-q^{m}\right) \sqrt{[m]_{q}}}+\frac{1}{2} \frac{1}{\sqrt{[m]_{q}}} \sqrt{\frac{1-q^{m+p}}{1-q^{m}}}\right)+1\right\} \\
& =\omega_{f}(\delta)\left\{\frac{1}{\delta}\left(\frac{1}{\sqrt{[m]}}\left(\frac{p}{1-q^{m}}+\frac{1}{2} \sqrt{\frac{1-q^{m+p}}{1-q^{m}}}\right)\right)+1\right\}
\end{aligned}
$$

Let $q=q_{m}$ satisfy $0<q_{m}<1, \lim _{m \rightarrow \infty} q_{m}=1$ and $\lim _{m \rightarrow \infty} q_{m}^{m}=c<1$.

Choosing $\delta=\delta_{m}=\left(\frac{1}{\sqrt{[m]}}\left(\frac{p}{1-q^{m}}+\frac{1}{2} \sqrt{\frac{1-q^{m+p}}{1-q^{m}}}\right)\right)^{m}$ as $\delta_{m} \rightarrow 0$ when $m \rightarrow \infty$ we obtain the desired result.

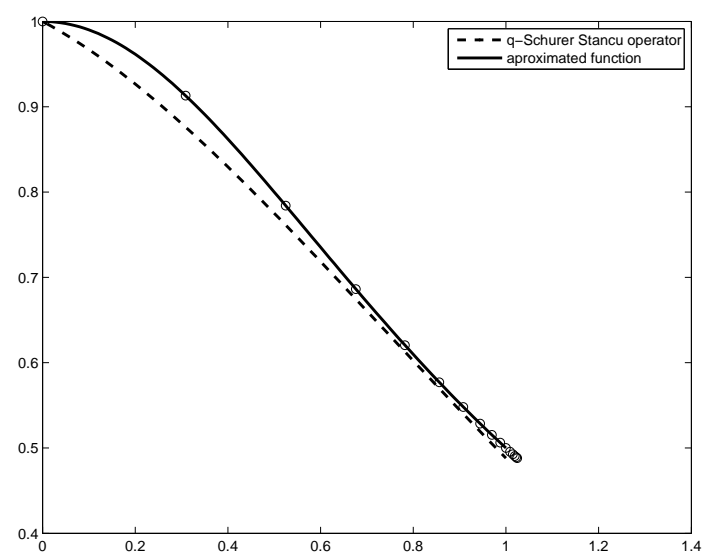

FigURE 1. The approximated function $f(x)=1 /\left(1+x^{2}\right), x \in$ $(0,1)$ with the $\mathrm{q}$-Schurer-Stancu operator, $\mathrm{q}=0.7 \alpha=1 / 40, \beta=1 / 6$, $m=10$ 


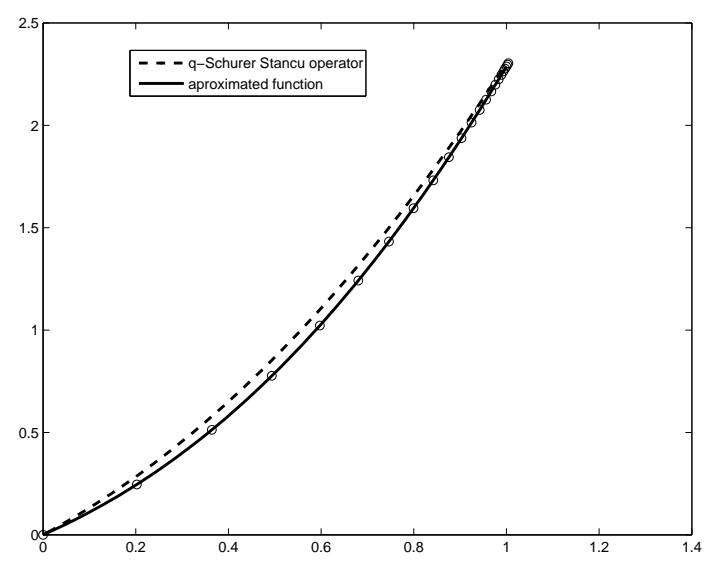

FIGURE 2. The approximated function $f(x)=\sin (x) e^{x}$ with the $\mathrm{q}$-Schurer-Stancu operator, $x \in(0,1), \mathrm{q}=0.8, \alpha=1 / 40, \beta=1 / 6$, $m=20$

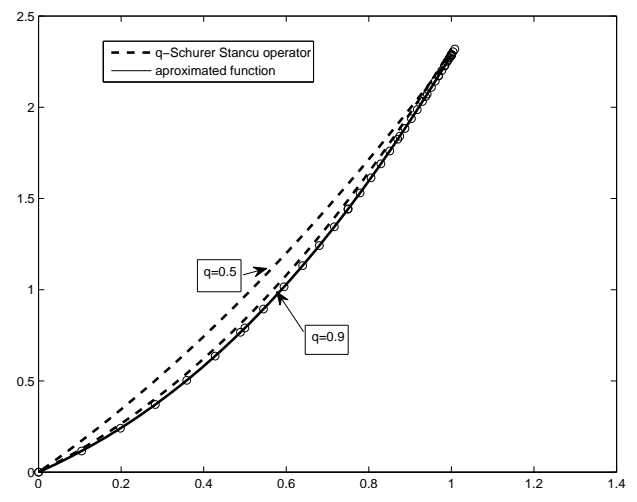

FIGURE 3 .

In the following we give some numerical results regarding the approximation properties of q-Schurer-Stancu operators using Matlab algorithms for construction of operators.

In Fig. 3 we notice that for the same function, when $0<\mathrm{q}<1$ is increasing, the curve of operator is closer, as follows from the convergence properties of operators given in Section 2. We notice also that for $0<\alpha, \beta<1$ the operator is interpolator on ends.

\section{REFERENCES}

[1] O. Agratini, "On statistical approximation in spaces of continuous functions," Positivity, vol. 13, no. 4, pp. 735-743, 2009. 
[2] F. Altomare and M. Campiti, Korovkin-type approximation theory and its applications, ser. de Gruyter Studies in Mathematics. Berlin: Walter de Gruyter, 1994, vol. 17.

[3] A. Aral and O. Doğru, "Bleimann, Butzer, and Hahn operators based on the $q$-integers," J. Inequal. Appl., vol. 2007, p. 12, 2007.

[4] O. Doğru and O. Duman, "Statistical approximation of Meyer-König and Zeller operators based on q-integers," Publ. Math., vol. 68, no. 1-2, pp. 199-214, 2006.

[5] O. Doğru and V. Gupta, "Monotonicity and the asymptotic estimate of Bleimann Butzer and Hahn operators based on $q$-integers," Georgian Math. J., vol. 12, no. 3, pp. 415-422, 2005.

[6] O. Doğru and V. Gupta, "Korovkin-type approximation properties of bivariate $q$-Meyer-König and Zeller operators," Calcolo, vol. 43, no. 1, pp. 51-63, 2006.

[7] O. Doğru and C. Muraru, "Statistical approximation by Stancu type bivariate generalization of Meyer-König and Zeller type operators," Math. Comput. Modelling, vol. 48, no. 5-6, pp. 961-968, 2008.

[8] V. Gupta and Z. Finta, "On certain $q$-Durrmeyer type operators," Appl. Math. Comput., vol. 209, no. 2, pp. 415-420, 2009.

[9] V. Gupta and W. Heping, "The rate of convergence of $q$-Durrmeyer operators for $0<q<1$," Math. Methods Appl. Sci., vol. 31, no. 16, pp. 1946-1955, 2008.

[10] V. Gupta and C. Radu, "Statistical approximation properties of $q$-Baskakov-Kantorovich operators," Cent. Eur. J. Math., vol. 7, no. 4, pp. 809-818, 2009.

[11] T. Trif, "Meyer-König and Zeller operators based on the $q$-integers," Rev. Anal. Numér. Théor. Approx., vol. 29, no. 2, pp. 221-229, 2000.

[12] M. C. V., "Note on $q$-Bernstein-Schurer operators," Studia UBB Mathematica, vol. LVI, no. 2, pp. $1-11,2011$

[13] H. Wang, "Properties of convergence for the $q$-Meyer-König and Zeller operators," J. Math. Anal. Appl., vol. 335, no. 2, pp. 1360-1373, 2007.

\section{Author's address}

\section{Carmen Violeta Muraru}

University "Vasile Alecsandri" of Bacau, Department of Mathematics and Informatics

E-mail address: carmen_7419@yahoo.com, cmuraru@ub.ro 